\title{
Análise da influência de parâmetros construtivos no funcionamento de um equipamento de infusão mecanizada
}

\author{
Analysis of the influence of construction parameters in the operation of a mechanical infusion \\ device
}

\author{
V. T. Bonow ${ }^{1 *}$; F. J. Macedo ${ }^{1}$; E. D. dos Santos ${ }^{1}$; F. B. dos Santos ${ }^{1}$; F. B. de \\ Mello $^{2} ;$ L. A. Isoldi ${ }^{1}$ \\ ${ }^{1}$ Escola de Engenharia (EE), Universidade Federal do Rio Grande (FURG), 96203-900, Rio Grande-RS, Brasil \\ ${ }^{2}$ Departamento de Engenharia, LIFEMED S/A, 96050-080, Pelotas-RS, Brasil \\ *viniciustim9@gmail.com \\ (Recebido em 14 de setembro de 2014; aceito em 29 de dezembro de 2014)
}

O presente trabalho possui como objetivo a caracterização de um problema encontrado em uma linha de produção de bombas de infusão peristáltica linear, com a utilização de um software capaz de interpretar dados experimentais por meio de análise estatística. O problema encontrado foi que ao se verificar a pressão máxima que o equipamento atingia, o mesmo não atendia os critérios de liberação do produto, o que ocasionaria uma possível vazão livre, podendo levar a óbito o paciente que por ventura viesse a utilizar o equipamento. Esta caracterização foi realizada através de experimentos, onde se verificou a influência de cada componente na pressão máxima do equipamento (refluxo), a qual existe uma especificação de um valor mínimo e máximo para a sua conformidade. Foram montadas várias configurações para o equipamento, variando uma peça específica e fixando as demais, obtendo assim várias respostas de pressão de refluxo. Após toda a coleta de dados, analisou-se, através do software de análise estatística MINITAB, os dados coletados, obtendo assim a peça mais crítica, onde devido a alguma variação desta, seria grande a influência na variável pressão de refluxo. Foi gerada uma reta de regressão com todos estes componentes e suas influências, tendo como resposta a pressão máxima da configuração inserida na reta. Com isso foi possível validar esta reta experimentalmente e analiticamente e redefinir as especificações de cada componente, considerando qualquer variação de lote de fornecimento ou tipo de peça, assegurando que o equipamento desenvolverá sua função de maneira segura e adequada.

Palavras-Chave: bomba de infusão, MINITAB, refluxo.

The present work aims to characterize a problem encountered in a production line of linear peristaltic infusion pumps, with the use of a software capable of interpreting experimental data by means of statistical analysis. The problem was verifying for the maximum pressure of equipment, which does not attempt the product specifications, which can cause a free flow and can lead to death of the patient that may eventually come to use the equipment. This characterization was performed through experiments where we evaluated the influence of each component in the maximum pressure in the system (reflux), which there is a specification of a minimum and maximum value for their compliance. Various settings for the equipment were assembled by varying a specific part and fixing the other, thus obtaining several reflux pressure responses. After all the data collection, analyzed by MINITAB statistical analysis software collected data, obtaining the most critical piece, where due to some variation of this would be a great influence in the variable pressure reflux. It was generated a regression line with all these components and their influences, and the maximum pressure in response inserted in the straight configuration. So, it was possible to experimentally validate this straight and analytically and reset the specifications of each component considering any variation in scope of delivery or type of piece, ensuring that equipment develop its function safely and appropriately.

Keywords: infusion pump, MINITAB, reflux.

\section{INTRODUÇÃO}

O emprego de bombas de infusão vem crescendo e cada vez mais equipamentos estão sendo lançados no mercado, agregando tecnologias e softwares capazes de interpretar dados vitais, críticos para o paciente, garantindo uma maior segurança ao mesmo. Equipamentos com softwares de alto controle referente à precisão de infusão tornaram-se uma necessidade básica, 
pois são amplamente utilizados em pacientes neonatal e também para a infusão de medicamentos que devem ter um extremo controle de dosagem, podendo levar a óbito o usuário devido a uma sobreinfusão ou subinfusão.

Existem três sistemas de infusão que podem ser empregados: o que utiliza controle manual de fluxo, e é o mais simples de todos; o que utiliza um controlador de infusão (automático ou semiautomático) para estabelecer o fluxo determinado pelo operador; e a bomba de infusão, que gera, monitora e controla o fluxo. A bomba de infusão, foco do presente trabalho, é o sistema que oferece a maior precisão de infusão, e permite trabalhar com pressões maiores que os sistemas gravitacionais. Nas bombas de infusão o controle do fluxo pode ser volumétrico ou não volumétrico [1].

Portanto a bomba de infusão é um equipamento eletromédico destinado a regular o fluxo de líquidos administrados ao paciente sob pressão positiva gerada pela bomba [2]. Um exemplo deste equipamento pode ser observado na Figura 1.

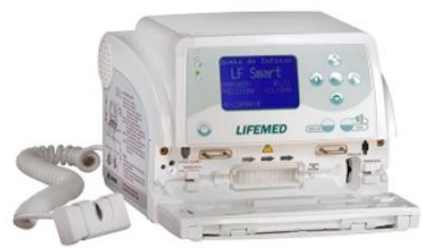

Figura 1: Bomba de infusão modelo LF Smart. Empresa LIFEMED S/A

De uma forma geral, existem dois tipos básicos de mecanismos de direcionamento para bomba de infusão: bomba de infusão peristáltica circular e bomba de infusão peristáltica linear. Estes dois tipos de bombas de infusão, apesar de ter o princípio de funcionamento muito semelhante, apresentam particularidades, que geram as características de cada bomba de infusão. Estes fatores, que serão detalhados à frente, são de extrema importância na escolha do tipo de bomba a se utilizar em determinados pacientes [3].

Nas bombas de infusão, a pressão positiva necessária para a administração do fluido é gerada através dos mecanismos de direcionamento. $\mathrm{O}$ mecanismo de direcionamento das bombas de infusão pode ser peristáltico (rotativo ou linear) ou no caso das bombas de infusão de seringa, emprega-se uma seringa (ou reservatório similar) com válvula associada a uma rosca sem fim que tem a finalidade de movimentar o êmbolo da seringa. Utiliza-se também um equipo do tipo pistão, que tem funcionamento semelhante à bomba de infusão de seringa [1].

O princípio de funcionamento peristáltico linear consiste em um esmagamento sequencial do tubo (equipo) sobre um batente, ocorrendo a formação da pressão positiva e assim havendo o deslocamento do fluido [4]. O movimento das hastes, chamadas comumente de "dedos" ocorre de forma ondulatória, realizando o deslocamento positivo necessário para que ocorra a infusão. Este mecanismo está representado na Figura 2. Para que este sistema funcione, é necessário um batente ao lado oposto dos dedos, pressionando o equipo. Este batente pode ser fixo, ou então, móvel.

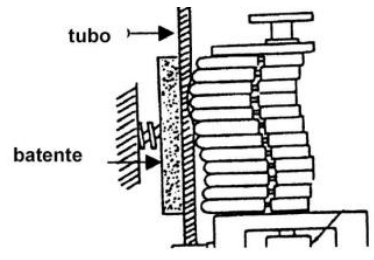

Figura 2: Mecanismo de infusão peristáltica linear

Desta forma, este trabalho abordará as características construtivas de um equipamento de infusão peristáltica linear, buscando um melhor desempenho no que diz respeito à precisão do mesmo. Para tal análise, serão avaliadas algumas respostas do equipamento no valor de refluxo (pressão máxima que o equipamento atinge), através da coleta de dados experimentais e posterior análise estatística através do software MINITAB, permitindo avaliar a influência de 
cada item considerado. Para isso, determinadas peças que compõem o sistema de infusão do equipamento foram estudadas, verificando sua influência na variável pressão de refluxo. São elas: o mecanismo peristáltico linear, a alavanca de fechamento da porta, o prensor, as molas e a chapa de fixação das molas. Cabe destacar que serão analisados aspectos construtivos dessas peças. Estes aspectos tratam tanto da parte de materiais, como também das características que cada componente deve possuir em função de sua aplicabilidade durante o uso do equipamento.

O mecanismo peristáltico linear é um parâmetro que influencia diretamente na pressão de refluxo e também na precisão do equipamento (volumetria). É a distância dos dedos em relação à face de contato com o batente (chamado aqui de prensor). Esta distância pode variar em aproximadamente $0,5 \mathrm{~mm}$ durante a montagem. Este mecanismo é apresentado na Figura 3.

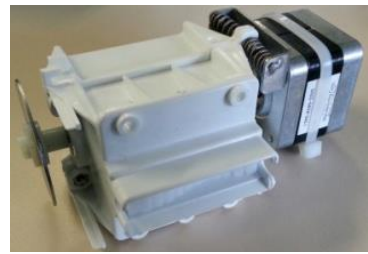

Figura 3: Mecanismo de infusão peristáltica linear - conjunto montado

A alavanca de fechamento da porta da bomba de infusão, apresentada na Figura 4, possui a função de manter a porta fechada durante a infusão do medicamento no paciente. Esta alavanca atualmente é produzida na própria empresa, que produz a bomba de infusão, pelo processo de injeção. O material de moldagem desta peça é a poliamida 6.6.

Sabe-se que a água atua como um plastificante causando um drástico efeito nas propriedades do material, reduzindo a rigidez e a estabilidade dimensional da poliamida 6.6 [5]. Com isto, a alavanca de fechamento da porta é um item com potencial de influência na pressão de refluxo, visto que esta possível variação de comportamento do material de projeto (poliamida 6.6) possa interferir no ideal desempenho do equipamento.

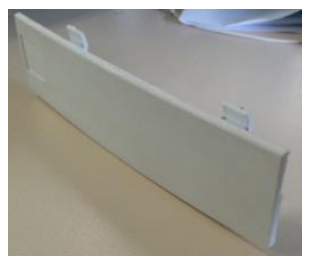

Figura 4: Indicação das travas de fechamento da alavanca

Já na Figura 5 é apresentado o prensor (batente do equipo a ser pressionado sobre os dedos do mecanismo peristáltico), em perspectiva. Como esta peça atua diretamente em contato com o equipo e mecanismo infusor, esta peça pode apresentar influência na pressão de refluxo do equipamento.

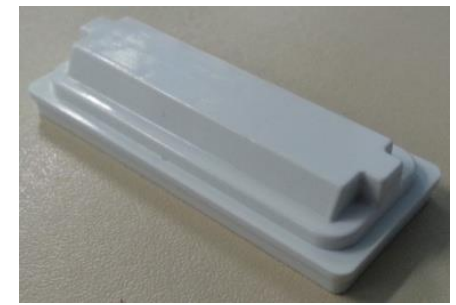

Figura 5: Prensor em perspectiva

O sistema de infusão é composto por cinco molas, posicionadas na região inferior do prensor, conforme indicado na Figura 6. Durante o processo de montagem, verificou-se que as tolerâncias de projeto adotadas estavam afetando o desempenho do equipamento, por isto este também é um item com potencial influência na pressão de refluxo. 


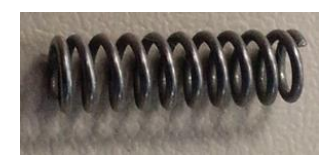

Figura 6: Mola do sistema de infusão

Por fim, a chapa de fixação das molas, mostrada na Figura 7, possui a função de manter as cinco molas alojadas no prensor. Esta peça possui cinco rebaixos, os quais possuem a função de alojar as molas. Apesar de não se ter tolerâncias e controle no recebimento desta medida, essa profundidade também afeta no valor de refluxo, pois conforme varia esta furação, a mola irá comprimir mais ou menos, influenciando na força de atuação do prensor e, consequentemente, variando na pressão de refluxo do equipamento.

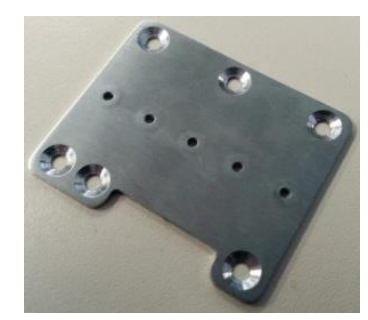

Figura 7: Vista em perspectiva da chapa de fixação das molas

\section{MATERIAL E MÉTODOS}

Através da metodologia descrita a seguir, foi possível correlacionar os parâmetros estudados, com a resposta na pressão de refluxo, podendo assim definir quais os parâmetros são mais relevantes para um ideal funcionamento do equipamento.

Resumidamente, gerou-se um arranjo ortogonal de Taguchi para a otimização dos experimentos, posteriormente, coletaram-se todos os valores das pressões de refluxo de cada configuração. Após toda a coleta, utilizou-se o software MINITAB para a interpretação de todos os dados, utilizando, por exemplo, ANOVA (analise de variância), gráficos de interação entre fatores, gráfico de resíduos, e geração de uma reta de regressão, essencial para reprojetar as peças consideradas críticas neste estudo, garantindo assim o ideal funcionamento do equipamento.

Então, para a otimização dos experimentos realizados, foi utilizado o método de Taguchi, que. O método de Taguchi consiste em uma ferramenta estatística que fornece uma forma sistemática e eficiente para otimizar o planejamento experimental através da redução do número de experimentos em relação ao arranjo fatorial completo [6].

Por esta razão Taguchi desenvolveu configurações especiais de experimentos fracionados, chamados de Arranjos Ortogonais (AO), que ajudam a determinar o número mínimo de experimentos para um determinado número de fatores [7].

Através desse método é possível planejar, conduzir e avaliar estatisticamente os experimentos, realizando um número mínimo de combinações, resultando em economia de tempo e recursos [8].

Então, para a avaliação experimental foi utilizada a metodologia de Taguchi, onde os cinco fatores em análise foram experimentados em dois níveis cada, os quais foram organizados em um arranjo L8, conforme apresentado na Tabela 1. Este arranjo foi gerado através do software MINITAB. 
Tabela 1: Arranjo experimental de Taguchi apresentando os fatores e seus níveis de avaliação para experimento 1 - pressão de refluxo

\begin{tabular}{cccccc}
\hline Experimentos & Molas & Prensor & $\begin{array}{c}\text { Distância entre } \\
\text { dedos e prensor }\end{array}$ & Chapa metálica \\
\hline $\mathbf{1}$ & 1 & 1 & 1 & 1 & 2 \\
\\
$\mathbf{2}$ & 1 & 1 & 1 & 1 & 2 \\
$\mathbf{4}$ & 1 & 2 & 2 & 2 & 1 \\
$\mathbf{5}$ & 1 & 2 & 2 & 2 & 2 \\
$\mathbf{6}$ & 2 & 1 & 2 & 2 & 1 \\
$\mathbf{7}$ & 2 & 1 & 1 & 2 & 1 \\
\hline
\end{tabular}

As molas foram caracterizadas quanto à medida de suas alturas, sendo que o nível 1 corresponde à um grupo de molas que apresentou altura média de $13,85 \mathrm{~mm}$ e o nível $2 \mathrm{um}$ grupo com altura de $14,82 \mathrm{~mm}$. As distâncias dos dedos do mecanismo peristáltico em relação à superfície do prensor foram consideradas de $5,70 \mathrm{~mm}$ e $5,30 \mathrm{~mm}$ para os níveis 1 e 2, respectivamente. Para a furação da chapa metálica foram considerados níveis 1 de $0,63 \mathrm{~mm}$ e 2 de $0,79 \mathrm{~mm}$. Para os materiais do prensor e da alavanca da porta, o poliacetal foi configurado como nível 1 (com valor numérico 1) e a poliamida 6 (prensor) e poliamida 6.6 (alavanca) como nível 2 (com valor numérico 2). A distribuição do experimento pode ser vista na Tabela 2.

Tabela 2: Arranjo experimental de Taguchi com o valor das variáveis

\begin{tabular}{cccccc}
\hline EXPERIMENTOS & $\begin{array}{c}\text { MOLAS } \\
(\mathbf{m m})\end{array}$ & $\begin{array}{c}\text { MATERIAL } \\
\text { DO PRENSOR }\end{array}$ & $\begin{array}{c}\text { DISTÂNCIA } \\
\text { ENTRE DEDOS E } \\
\text { PRENSOR }(\mathbf{m m})\end{array}$ & $\begin{array}{c}\text { CHAPA } \\
\text { METÁLICA }(\mathbf{m m})\end{array}$ & ALAVANCA \\
\hline $\mathbf{1}$ & 13,85 & Poliacetal & 6,85 & 0,628 & Poliacetal \\
$\mathbf{2}$ & 13,85 & Poliacetal & 6,85 & 0,786 & Poliamida 6.6 \\
$\mathbf{3}$ & 13,85 & Poliamida 6 & 6,45 & 0,628 & Poliacetal \\
$\mathbf{4}$ & 13,85 & Poliamida 6 & 6,45 & 0,786 & Poliamida 6.6 \\
$\mathbf{5}$ & 14,82 & Poliacetal & 6,45 & 0,628 & Poliamida 6.6 \\
$\mathbf{6}$ & 14,82 & Poliacetal & 6,45 & 0,786 & Poliacetal \\
$\mathbf{7}$ & 14,82 & Poliamida 6 & 6,85 & 0,628 & Poliamida 6.6 \\
$\mathbf{8}$ & 14,82 & Poliamida 6 & 6,85 & 0,786 & Poliacetal \\
\hline
\end{tabular}

A variável resposta deste experimento foi a pressão de refluxo da bomba, em psi, medida em um manômetro digital com resolução de 0,1 psi. Esta variável foi analisada no MINITAB quanto à influência dos fatores através da ANOVA (análise de variância), determinando-se os efeitos médios e as interações entre os fatores. Também, foi realizada uma análise de Regressão Linear para ajustar os dados experimentais encontrados. Todas as análises estatísticas foram realizadas utilizando-se o software MINITAB, com nível de significância de 95\%.

Para complementar o estudo, foi feita uma validação da reta de regressão, apresentada na Equação (1), através do comparativo entre novas medidas de refluxo e os valores estimados através da reta gerada pelo software MINITAB.

Para a obtenção do erro foi utilizada a seguinte equação:

$$
\text { Erro }(\%)=\left(1-\left(\frac{\text { pressão de refluxo calculada }}{\text { pressão de refluxo obtida }}\right)\right) \times 100
$$

Após esta validação, utilizou-se a mesma para reprojetar as peças de maior influência (mola, chapa de fixação e distância entre os dedos do mecanismo infusor e prensor), definindo assim uma nova especificação de projeto que garanta o ideal funcionamento do equipamento em qualquer configuração de montagem. 


\section{RESULTADOS E DISCUSSÃO}

Primeiramente, conforme a metodologia e o arranjo experimental definido pelo método de Taguchi (ver Tabelas 1 e 2), gerado pelo MINITAB, foi avaliada cada peça, em seus extremos de configuração, onde foram coletadas 3 medidas para cada configuração.

Na Tabela 3 é possível verificar as oito configurações com as três medidas coletadas da pressão de refluxo.

Tabela 3: Medições conforme arranjo ortogonal de Taguchi

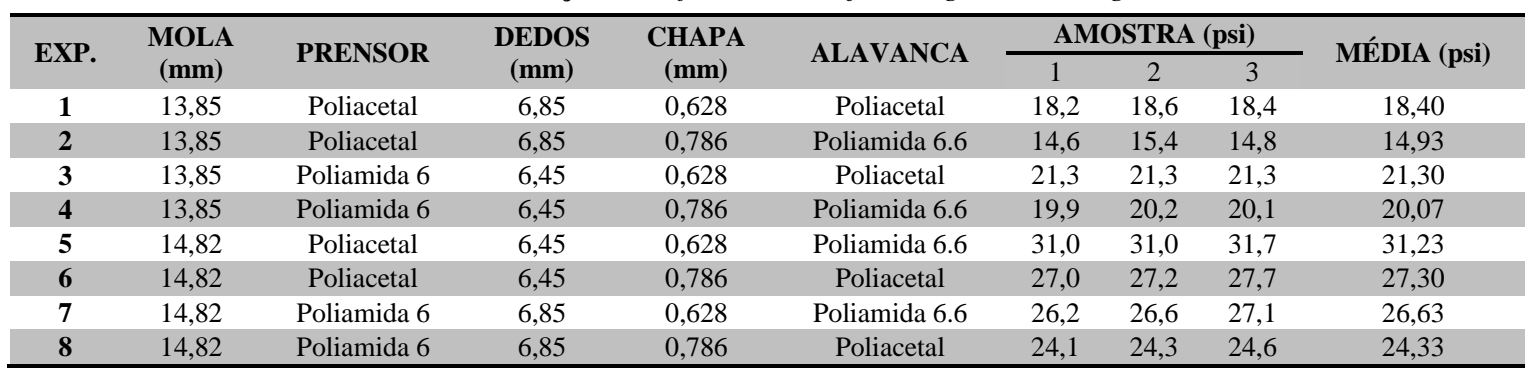

Na Figura 8 é possível verificar as respostas da pressão de refluxo em função do experimento realizado, mostrando as diferenças existentes entre cada configuração (média de casa amostra).

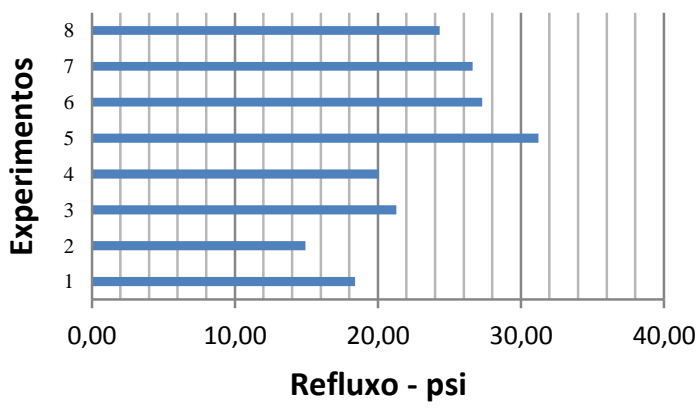

Figura 8: Valores médios da pressão de refluxo (psi)

Após os valores coletados, foi utilizado o MINITAB para a avaliação da influência de cada fator isolado na pressão de refluxo do equipamento, bem como para a avaliação das interações entre diferentes níveis de fatores.

A Figura 9 apresenta os efeitos médios de cada um dos fatores estudados com relação a resposta do refluxo do equipamento obtida através do software.

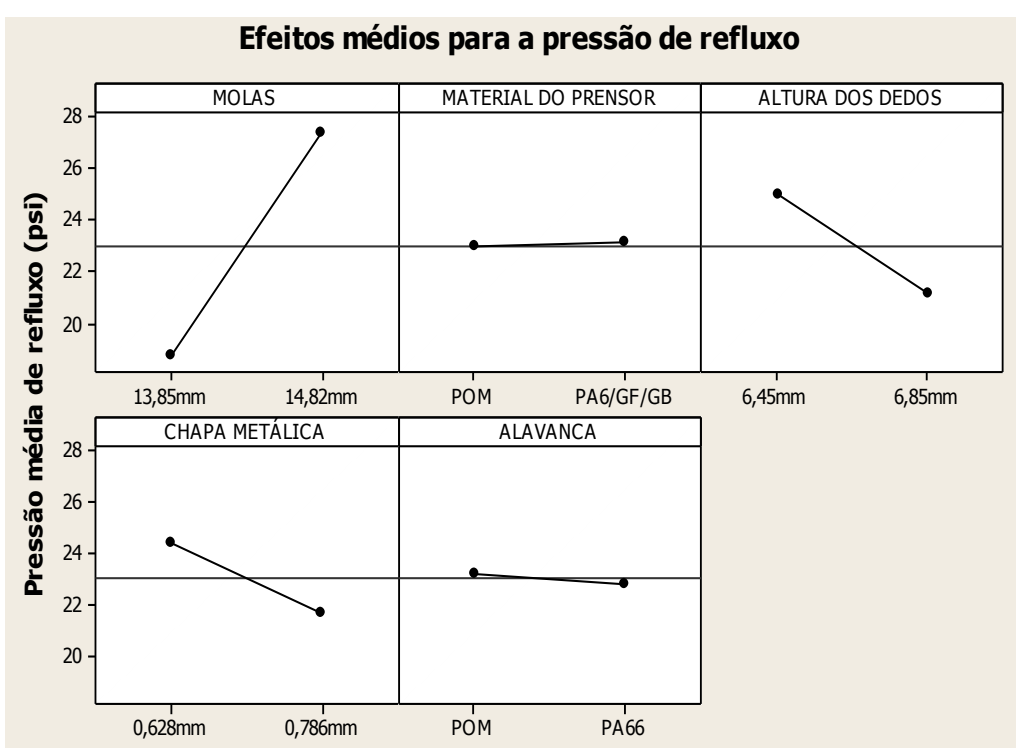


Figura 9: Efeitos médios dos fatores na pressão de refluxo da bomba LF Smart

Os efeitos principais que afetam significativamente a pressão de refluxo do equipamento são a altura das molas, a distância dos dedos do mecanismo peristáltico em relação à superfície do prensor e a profundidade do alojamento das moldas na chapa metálica, como pode ser observado na Figura 9. Isto pode ser comprovado numericamente através da estatística $\mathrm{F}$ da análise de variância apresentada na Tabela 4, gerada através do software MINITAB.

Tabela 4: Análise de Variância para influência dos fatores na pressão de refluxo da bomba LF Smart

\begin{tabular}{|c|c|c|c|c|}
\hline PEÇA & Graus de Liberdade & Soma dos Quadrados & Média Quadrática & $\mathbf{F}$ \\
\hline MOLAS & 1 & 454,14 & 454,14 & 1114,45 \\
\hline PRENSOR & 1 & 0,08 & 0,08 & 0,20 \\
\hline DEDOS & 1 & 91,26 & 91,26 & 223,95 \\
\hline ALAVANCA & 1 & 0,88 & 0,88 & 2,16 \\
\hline Erro & 18 & 7,34 & 0,41 & \\
\hline Total & 23 & 598,53 & & \\
\hline
\end{tabular}

Pela análise mostrada na Tabela 4, estes três fatores (altura das molas, distância dos dedos do mecanismo peristáltico em relação a face do prensor e profundidade do alojamento das moldas) são os que afetam significativamente a pressão de refluxo da bomba, pois apresentam valores da estatística $\mathrm{F}$ acima do $\mathrm{F}$ crítico tabelado de 4,414.

A Figura 10, gerada no software MINITAB, apresenta a interação entre os fatores envolvidos no experimento de pressão de refluxo da bomba LF Smart, estudada no presente trabalho. Para a altura das molas não foi encontrado nenhum tipo de interação com os demais fatores envolvidos no experimento, indicando que esta variável corresponde ao fator principal desta variação. Também, destaca-se que molas maiores (linhas vermelhas tracejadas) resultam em pressão de refluxo maior do que molas menores (linhas pretas).

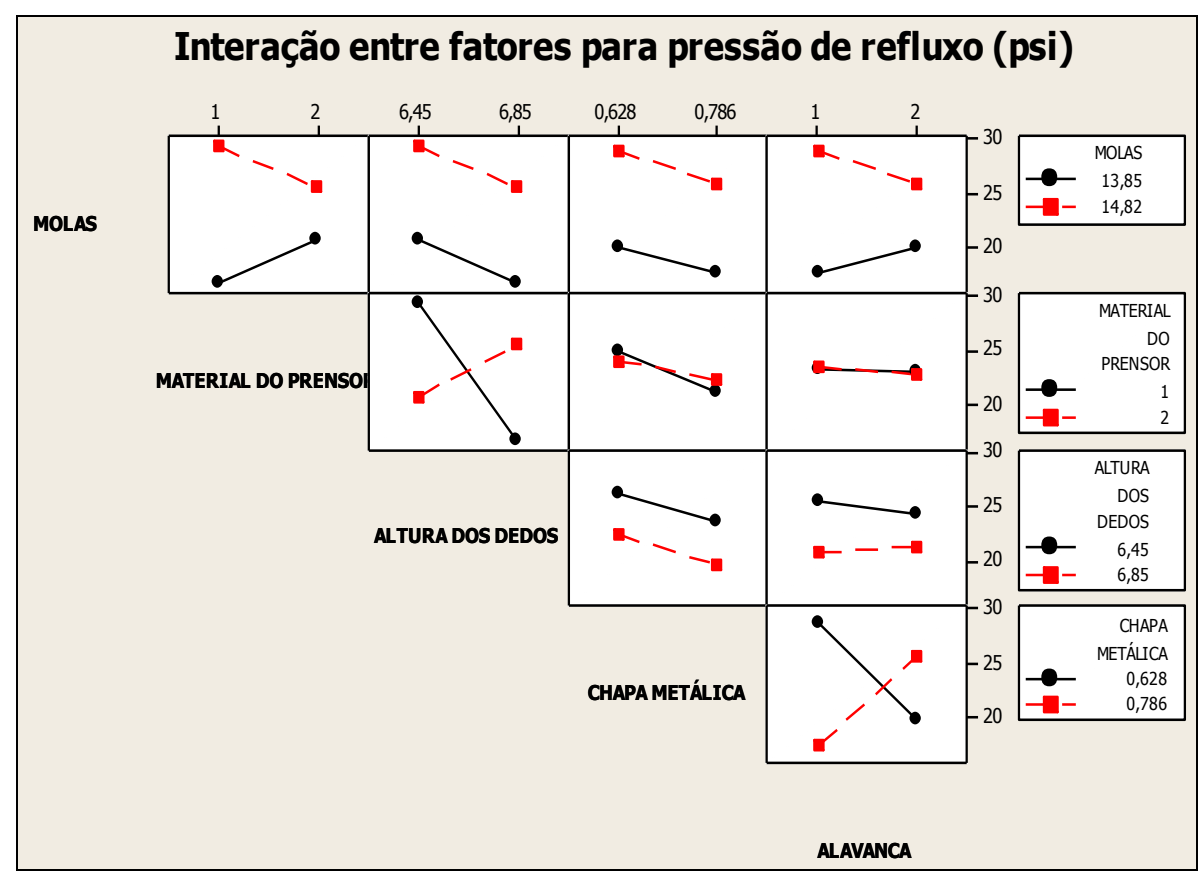

Figura 10: Interação entre os fatores no experimento de pressão de refluxo da bomba LF Smart

A Figura 10 indica ainda que para o material do prensor foi encontrada interação inversa com a distância entre os dedos do mecanismo peristáltico em relação à superfície do prensor. Já para o prensor em poliamida 6 o efeito foi contrário, embora em menor escala. Interação similar foi encontrada também para os fatores profundidade do alojamento da mola e material da alavanca da porta. Estas interações destacam a influência dos fatores distância do mecanismo 
peristáltico em relação à superfície do prensor e da profundidade do alojamento da mola na pressão de refluxo da bomba, conforme determinado pela Análise de Variância.

A Figura 11 apresenta a probabilidade normal para resíduos, obtida através do software MINITAB, indicando que os dados comportam-se segundo esta distribuição, validando desta forma a análise estatística baseada em uma distribuição normal dos dados, correlacionando os dados obtidos no experimento. Desta forma não foram encontradas evidências de não normalidade acentuada.

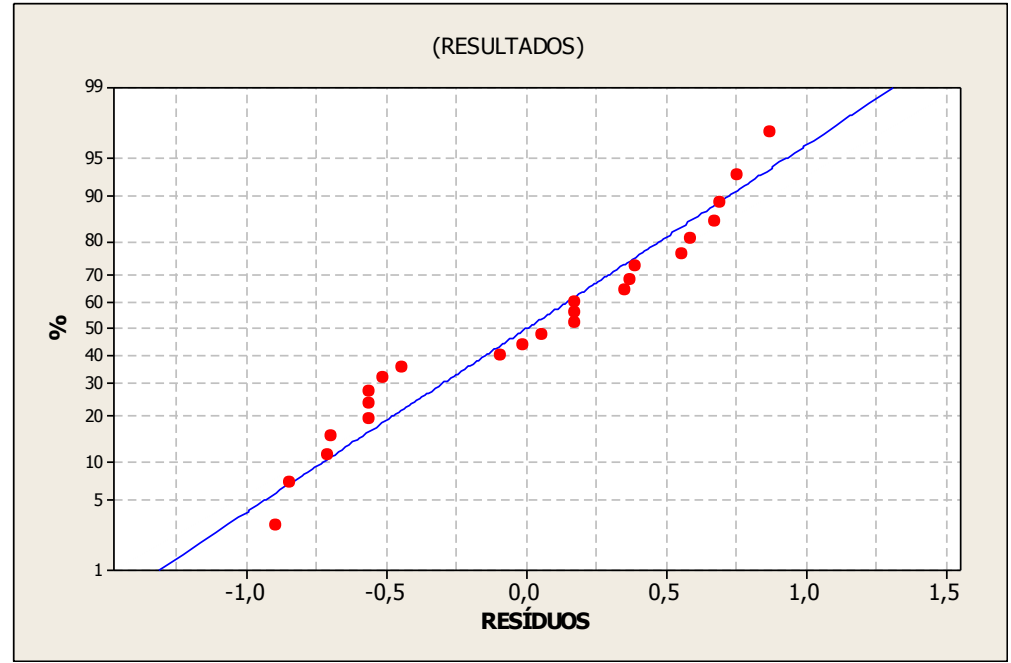

Figura 11: Gráfico de probabilidade normal para resíduos

Conforme dados da Figura 11, que validam a análise estatística, foi possível gerar uma reta de regressão através do MINITAB, com os fatores estudados, podendo-se prever a pressão de refluxo do equipamento de acordo com a configuração de peças montadas.

A Tabela 5 apresenta os resultados da Análise de Regressão para os fatores avaliados.

Tabela 5: Análise de Regressão da pressão de refluxo da bomba sobre os fatores

\begin{tabular}{cc}
\hline Preditor & Coeficiente \\
\hline Constantes & $-28,0780$ \\
MOLAS & 8,9691 \\
PRENSOR & 0,1167 \\
DEDOS & $-9,7500$ \\
CHAPA & $-17,3000$ \\
ALAVANCA & $-0,3833$ \\
\hline
\end{tabular}

Para esta análise foi encontrado um valor de $\mathrm{R}^{2}$ de 0,984 , indicando um ótimo ajuste linear positivo entre os dados experimentais. A seguir é apresentada a equação de regressão obtida para a pressão de refluxo $(\mathrm{P})$ com este ajuste de dados:

$$
\mathrm{P}=8,97 \alpha-9,75 \beta-17,3 \gamma+0,117 \delta-0,383 \varepsilon-28,08
$$

onde $\alpha$ é altura média das molas; $\beta$ é distância entre dedos e prensor somada à espessura da chapa de apoio; $\gamma$ é a profundidade do alojamento das molas na chapa metálica; $\delta$ é o material do prensor, que tem valor 1 na Equação (2); e $\varepsilon$ é o material da alavanca da porta, que tem valor 2 na Equação (2).

Em função dos dados obtidos pela análise dos experimentos na estatística $\mathrm{F}$, o material do prensor e o material da alavanca não representam influência significativa na resposta da pressão de refluxo, por isto a Equação (2) pode ser ajustada em função das constantes associadas ao material do prensor e da alavanca, resultando em:

$$
\mathrm{P}=8,97 \alpha-9,75 \beta-17,3 \gamma-28,73
$$

Com a Equação (3) é possível prever o comportamento da pressão de refluxo da bomba em função da variação dos fatores estudados. 
Esta variação na pressão de refluxo em função da variação em três características dimensionais de componentes de montagem da bomba corrobora com as observações práticas realizadas nos experimentos, onde as pressões de refluxo variavam entre 7,0 e 23,0 psi entre bombas montadas de acordo com o processo normal especificado pelo projeto do equipamento. Desta forma, através da equação, é possível determinar em um caso real, qual é ou quais são os parâmetros que devem ser alterados em caso de retrabalho de uma bomba.

Assim, através dos dados e resultados obtidos, pode-se notar que os fatores que mais influenciam na pressão do refluxo do equipamento foram as molas, a chapa de fixação das molas e a distância dos dedos do mecanismo em relação à face do prensor.

Utilizando a Equação (3), chegou-se a um conceito de peças em que qualquer configuração de montagem o refluxo permanecerá dentro da faixa aceitável para a liberação e garantia de que o equipamento esteja conforme.

Na Tabela 6 é mostrado uma coleta de dados (dimensões de mola, chapa e distância dos dedos em relação ao prensor) de 3 equipamentos e comparado com o valor calculado através da Equação (3), utilizando o software Excel para este cálculo.

Tabela 6: Comparação entre refluxo calculado e real

\begin{tabular}{|c|c|c|c|c|c|}
\hline & Mola média (mm) & Prof. Chapa (mm) & Mecanismo (mm) & & \\
\hline \multirow{2}{*}{1} & 14,838 & 0,722 & 6,83 & & \\
\hline & Refluxo Calculado & & 25,3 & psi & Erro \\
\hline \multirow{3}{*}{2} & Mola média $(\mathrm{mm})$ & Prof. Chapa (mm) & Mecanismo (mm) & & \\
\hline & 14,670 & 0,906 & 7,00 & & \\
\hline & Refluxo Calculado & & 18,9 & psi & Erro \\
\hline \multirow{4}{*}{3} & Mola média $(\mathrm{mm})$ & Prof. Chapa (mm) & Mecanismo (mm) & & \\
\hline & 14,438 & 1,092 & 6,84 & & \\
\hline & Refluxo Calculado & & 15,2 & psi & Erro \\
\hline & Refluxo encontrado & & 14,9 & psi & $-2,00 \%$ \\
\hline
\end{tabular}

Conforme a Tabela 6, o erro encontrado foi menor que 4\%, para mais e para menos. Esta diferença, provavelmente, está relacionada a dificuldades e variações de medição da mola e também devido a adequação da curva da reta de regressão para a geração da equação utilizada para o cálculo do refluxo $\left(\mathrm{R}^{2}=0,984\right)$.

Para o redimensionamento dos itens, foi considerado que o processo de fabricação da mola possui uma maior variação dimensional entre as peças, onde se reduziu a tolerância da chapa, para poder dar mais liberdade dimensional às molas.

Na Tabela 7 é apresentado o resultado de tolerância adotado para as peças e a resposta de refluxo calculada através da Equação (3).

Tabela 7: Definição de tolerância para as peças de influência no refluxo

\begin{tabular}{ccccc}
\hline & MECANISMO & MOLA & CHAPA & REFLUXO \\
\hline TOLERÂNCIA INFERIOR & $5,75 \mathrm{~mm}$ & $14,55 \mathrm{~mm}$ & $0,95 \mathrm{~mm}$ & $18,1 \mathrm{psi}$ \\
TOLERÂNCIA SUPERIOR & $5,55 \mathrm{~mm}$ & $14,8 \mathrm{~mm}$ & $0,85 \mathrm{~mm}$ & $24,0 \mathrm{psi}$ \\
\hline
\end{tabular}

Para que as tolerâncias da Tabela 7 sejam cumpridas, é importante que seja adotado um rigoroso critério de verificação de recebimento das peças (mola e chapa de fixação das molas), assim como um rigoroso controle no processo de montagem do equipamento, garantindo que as medidas de mecanismo infusor (medidas do mecanismo infusor da tabela acima desconsidera a chapa de auxílio na medição) estejam dentro do especificado.

\section{CONCLUSÃO}

A partir do presente trabalho conclui-se que, após todos os testes realizados, e com o auxílio do software MINITAB (sendo este de essencial uso, pois sem ele não seria possível à geração dos Arranjos Ortogonais e também a interpretação dos dados), pode-se concluir sobre a influência dos parâmetros construtivos do equipamento, que: 
- Dos fatores analisados, os que mais influenciaram na pressão de refluxo foram as molas, a chapa de fixação das molas e a distância do mecanismo infusor (ANOVA);

- Os fatores alavanca e prensor tiveram a influência desprezada devido ao baixo valor da estatística F calculada;

- Através da Equação (3) gerada para a determinação da pressão de refluxo, pode-se chegar a um valor muito próximo do valor encontrado na prática, o que valida esta equação;

- Após o redimensionamento das peças que influenciavam na pressão de refluxo, e anterior reprovação na linha de produção de aproximadamente $85 \%$, chegou-se a um equipamento em seu desempenho ideal, onde após a implementação destes novos fatores, o medicamento é liberado $100 \%$ sem nenhum problema de refluxo.

\section{AGRADECIMENTOS}

Os autores agradecem à LIFEMED e ao CNPq pelo apoio.

\section{REFERÊNCIAS BIBLIOGRÁFICAS}

1. Button VLSN. Dispositivos de Infusão. [Acesso em: 28/04/2014]. Disponível em: http://www.deb.fee.unicamp.br/vera/bombadeinfusao.pdf

2. Associação Brasileira de Normas Técnicas (ABNT). Equipamento eletromédico - Parte 1 Prescrições particulares para segurança de bombas e controladores de infusão. Rio de Janeiro. Norma NBR IEC 60601-2-24; 1999.

3. Silva ÁMJr. Sistema para avaliação da funcionalidade de bombas de infusão [dissertação]. Florianópolis (SC): Universidade Federal de Santa Catarina (UFCS); 2002. 110 p.

4. Alves MAC. Bombas de infusão: operação, funcionalidade e segurança [dissertação]. Florianópolis (SC): Universidade Federal de Santa Catarina (UFSC); 2002. 109 p.

5. Kutz M. Handbook of materials selection. New York: John Wiley \& Sons; 2002. 1520 p.

6. Souza HJC, Moyses CB, Pontes FJ, Duarte RN, Silva CES, Alberto FL, Ferreira UR, Silva MB. Optimization molecular assay optimized by Taguchi experimental design method for venous thromboembolism investigation. Mol Cell Probes. 2011 Oct-Dec;25(5-6):231-7, doi:10.1016/j.mcp.2011.08.001

7. Roy RK. A primer on the Taguchi method. New York: Van Nostrand Reinhold; 2010.

8. Lira C, Alarcon OE. Seleção de esmaltes cerâmicos utilizando um delineamento experimental segundo Taguchi. Cerâmica. 2004 Oct-Dec;50(316)308-17, http://dx.doi.org/10.1590/S036669132004000400005 